\title{
The consumption of a saccharin-glucose solution by satiated and food-deprived immature rats'
}

JAN W. KAKOLEWSKI, VERNE C. COX AND ELLIOT S. VALENSTEIN, FELS RESEARCH INSTITUTE, Yellow Springs, Ohio

Immature rats consumed a combined saccharintglucose solution in large quantities comparable to that consumed by adult animals. However. during periods of food deprivation immature animals consumed relatively more of this solution than mature animals. These results are related to the development of antidiuretic activity.

The authors (Valenstein, Cox, \& Kakolewski, 1967b) had previously shown that mature rats consume large quantities of a solution of saccharin plus glucose $(S+G)$. Food-satiated animals consumed the $S+G$ solution in quantities approximating $55 \%$ of their body weight during $24 \mathrm{~h}$ periods and displayed no tendency to reduce their intake over successive days. If deprived of food, the animals gradually increased their intake to approximately $100 \%$ of their body weight. As animals given equivalent glucose and saccharin solutions in separate bottles consume less than $40 \%$ of the fluid intake of the $S+G$ mixture, we concluded that our results represented a type of polydipsia elicited by the synergistic action of the two substances. Recently, Hsiao \& Rasmussen (1968) used a different strain of mature rats and reported that our $S+G$ solution was consumed in larger quantities than other sweet solutions tested. Earlier, Sheffield, Roby, \& Campbell (1954) used a similar mixture of saccharin and glucose and reported that this was a more effective reward for mature rats than either a glucose or saccharin solution judged both by running speed and amount consumed in the brief period in the goal box of a runway. Recently, Phillips, Morgan, \& Mogenson (1968) reported that our $S+G$ solution was the only solution tested to be preferred over reinforcing brain stimulation in a competitive situation.

In the present experiment we determined if immature rats exhibit a comparable high intake of the $S+G$ solution and if these young animals also increase their consumption of this solution when deprived of food. Lastly, we had previously raised the possibility that the palatability of the $S+G$ solution induces excessive drinking, resulting in a plasma volume increase triggering an inhibition of antidiuretic, hormone (ADH). The study of Heller (1960), which demonstrated that the kidneys of young rats do not concentrate urine in the same way as they do in adults and that the presence of $\mathrm{ADH}$ cannot be detected in young animals, provided an additional impetus for the present study. Consequently, we wished to determine if immature animals would consume even more $S+G$ solution relative to body weight than do adult animals.

Method. ${ }^{2}$ Twenty-three albino rats ( 12 male, 11 female) of the Holtzman strain were used. The average age at the start of the 16-day experiment was 25.5 (range: 24-27) and the average body weight at this time was $58 \mathrm{~g}$ (range: 39-83). The animals were housed in individual cages and had no experience with sweet solutions prior to testing. Body weight and food (when available) and fluid consumption were measured daily. Food consisted of Purina lab chow pellets. The solutions available were tap water and/or a $S+G$ mixture of $0.125 \mathrm{~g}$ of sodium saccharin (sodium-o-benzoic sulfimide) and $3.0 \mathrm{~g}$ of glucuse dissolved in distilled water to a total volume of $100 \mathrm{ml}$. Every day, drinking bottles were washed and solutions that had been stored for $24 \mathrm{~h}$ under refrigeration were provided. Fluid spilled was captured in plastic cylinders mounted under each drinking tube.

Food was available ad lib except for Days 11-14 when the animals were completely deprived of food. Baseline consumption of tap water and food were obtained for the initial seven days; thereafter, the $S+G$ solution and the tap water were both available.

Results and Discussion. Figure 1 summarizes the results. During the first three days that both the $S+G$ solution and the tap water were available (Days 8-11) the animals drank the $S+G$ solution in quantities equal to about $50 \%$ of their body weight. Immediately after the $S+G$ solution was made available the animals essentially ignored the tap water, consuming only negligible quantities. With food available ad lib the animals consumed approximately $2-1 / 2$ times more of the $S+G$ solution than they had been consuming of the tap water when it was the only fluid available. During the following four days, when the animals were without food, the consumption of the $S+G$ solution increased rapidly and monotonically, reaching a maximum of $165 \mathrm{ml}$ during the fourth fooddeprived day. This consumption was equal to $217 \%$ of the depressed body weight of the animals on that day and over $150 \%$ of the highest body weight achieved by the animals. On the last two days when food was provided again, the consumption of the $S+G$ solution declined precipitously, but remained above $70 \%$ of the body weight.

An analysis of the consumption of the $S+G$ solution revealed that females consumed significantly more $(p<.005$, Wilcoxon test) relative to body weight than males (females $1.00 \mathrm{ml} / 1.53 \mathrm{~g}$ body weight; males $1.00 \mathrm{ml} / 2$ body weight). These findings may be relevant to the recent reports of sex differences in the consumption of sweet solutions (Valenstein, Cox, \& Kakolewski, 1967a; Valenstein, Kakolewski, \& Cox, 1967; Wade \& Zucker, 1968).

The results indicate clearly that immature rats, as well as mature animals, consume large quantities of an $S+G$ solution and when deprived of food the young animals display an even more dramatic increase in consumption of this solution. In this context it may be

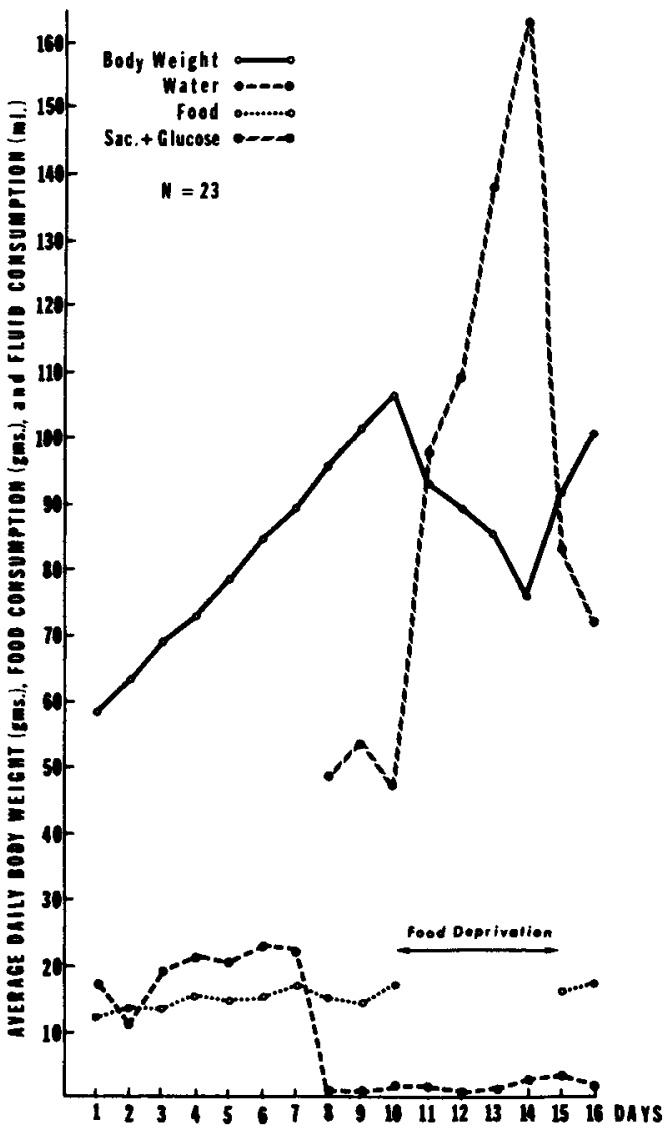

Fig. 1. 
important to note that the younger females in our sample consumed significantly more of the $S+G$ solution than the older animals. A similar difference was not observed in the male animals. It is possible that the low levels of ADH reported to be present in the plasma of young rats may permit a more extreme diuresis to better compensate for the intake of large quantities of fluid when deprived of food. In our laboratory, we have found that providing the $S+G$ solution, particularly in the absence of food, is a most convenient way to have animals load themselves with large amounts of fluids. This method avoids the stress involved in imposing gastric loads upon the animals.

\section{REFERENCES}

HELLER, J. The physiology of the antidiuretic hormone. V. The antidiuretic activity of rat plasma during ontogeny. Physiol Bohem. (Prague), 1960, 9, 289-293.

HSIAO, S., \& RASMUSSEN, C. Why does a variety of measures of thirst yield different results? An explanation developed by studying the effect of an aversive factor on liquid intake in rats. Psychon. Sci, 1968, 10, 97-98.

PHILLIPS, A. G., MORGAN, C. W., \& MOGENSON, G. J. Brain stimulation versus water, saccharin or sucrose in food and water deprived rats.
Presented at the Eastern Psychological Association meeting, April 8, 1968. SHEFFIELD, F. D., ROBY, T. B., \& CAMPBELL, B. A. Drive reduction versus consummatory behavior as determinants of reinforcement. J. comp. physiol Psychol, 1954, 47, 349-354.

VALENSTEIN, E. S., COX, V. C., \& KAKOLEWSKI, J. W. Further studies of sex differences in taste preferences with sweet solutions. Psychol Rep., 1967a, 20, 1231-1234.

VALENSTEIN, E. S., COX, V. C., \& KAKOLEWSKI, J. W. Polydipsia elicited by the synergistic action of a saccharin and glucose solution. Science, 1967b, 157, 552-554.

VALENSTEIN, E. S., KAKOLEWSKI, J. W., \& COX, V. C. Sex difference in taste preference for glucose and saccharin solutions. Science, 1967, 156, 942-943.

WADE, G., \& ZUCKER, I. Personal communication and presented at the Western Psychological Association meeting (1968) by I. Zucker.

\section{NOTES}

1. Supported by NIMH Grant M-4529, Career Scientist Award MH-4947 and NASA Research Grant NsG-437.

2. The authors would like to acknowledge the competent assistance of Antioch College students, Peter Beal and Nancy Maher. 